\title{
Calidad del aire en Quibdó mediante bioindicadores
}

\section{Air quality of the town of Quibdó using bioindicators}

\section{Rosmary Mena*}

\section{Resumen}

El uso de bioindicadores para monitorear la calidad del aire de una zona específica permite evaluar alteraciones medioambientales asociadas con la contaminación atmosférica. El crecimiento de la parte urbana del municipio de Quibdó y de su parque automotor, trajo como consecuencia el incremento de la contaminación. Mediante el uso de bioindicadores (epífitos) se valoró la calidad del aire en diferentes estaciones del área de estudio. Se caracterizaron los índices de pureza atmosférica (IPA) en el casco urbano de Quibdó y se sectorizó en cinco zonas diferenciadas de calidad del aire, siendo las estaciones ubicadas en el centro de la ciudad las que presentan la contaminación máxima, con un rango de valores IPA entre 5,3 y 9,5. La estación menos contaminada se encuentra ubicada en la zona norte de la ciudad, donde se presenta una baja intervención antrópica y escaso tráfico vehicular y su IPA, el máximo obtenido, fue 58,5. Se determinaron 74 especies de epifitos, de los cuales se diferenciaron 34 líquenes costrosos (45,9\%), 9 líquenes foliosos (10,8\%) y 1 fruticoso $(1,4 \%)$. Además se identificaron 13 tipos de musgos equivalentes al 17,6\%, 14 especies de hepáticas pertenecientes a la familia Legeuneaceae, correspondiente a 18,9\% de los especímenes y en 5,4\% de las muestras micelios de hongos. La zonificación obtenida es la primera realizada de acuerdo con este criterio en el municipio, logrando aportar a las autoridades ambientales un insumo para la toma de decisiones y en el desarrollo de actividades de prevención, monitoreo y control en este tema.

Palabras clave: Calidad del aire, Bioindicadores, Líquenes, Zonificación.
* Ingeniera ambiental. Especialista Gerencia de los Recursos Naturales. Contratista Instituto de Investigaciones Ambientales del Pacífico (IIAP), Quibdó, Colombia.e-mail: rosmarymm@hotmail.com

Recibido: 9 de febrero de 2012

Aceptado: 2 de abril de 2012

\begin{abstract}
The use of biomarkers to monitor air quality in a specific area to evaluate environmental changes associated with air pollution. The growth of the urban part of the municipality of Quibdó and its fleet resulted in increased pollution. By using bioindicators ( epiphytes ) assessed the air quality in different stations of the study area. Characterized atmospheric purity indices (API) in the town of Quibdó and zoning in five areas differentiated air quality stations being located in the center City those having the highest contamination, with a range of API values between 5.3 and 9.5. The station is less contaminated located in the north of the city, which has a low intervention anthropic and scarce vehicular traffic and API, the maximum obtained was 58.5. It epifitos 74 species identified, of which 34 were differentiated lichens crusty (45.9\%), 9 foliose lichens (10.8\%) and 1 fruticose (1.4\% ). Addition identified 13 types of mosses equivalent to $17.6 \%$, 14 species of liverworts Legeuneaceae belonging to the family corresponding to $18.9 \%$ specimens and $5.4 \%$ of fungal mycelia samples. Zoning obtained is the first carried out according to this criterion in the municipality, achieving environmental authorities provide input for decision
\end{abstract}




\section{Bioetnia Volumen 9 № 2 (julio-diciembre), 2012}

-making and the development of prevention, monitoring, control on this issue.

Keywords: Air quality, Biomarkers, Lichens, Zoning.

\section{Introducción}

Desde hace décadas es una preocupación de todos los países, la necesidad de implementar estudios de evaluación, monitoreo y control, a los fenómenos y cambios ambientales y ecológicos, causados por la actividades humanas para evaluar las alteraciones y definir estrategias de recuperación y remediación. Aún más, detectar a tiempo los daños y tomar medidas preventivas.

La calidad del aire está determinada por la composición y concentración de los gases presentes en la capa de la ecosfera y de su toxicidad para los seres vivos. Dicha calidad es determinante para la salud pública, la morbilidad y mortalidad concerniente a enfermedades respiratorias en la población, la cual está directamente relacionada con la contaminación atmosférica y por esto el aire que respiramos constituye un capital ecológico importante que vale la pena cuidar.

Durante las últimas décadas el aumento del parque automotor en las ciudades, el consumo de combustibles fósiles en diferentes actividades industriales y semiindustriales entre otras, ha incrementado la emisión de gases efecto invernadero y problemas como el cambio climático global, trayendo como consecuencias directas afectaciones a la salud y calidad de vida de la población, independiente de su ubicación geográfica.

De acuerdo con O'Neill et al. (1992), el parque automotor en el país es el responsable del mayor porcentaje de gases contaminantes en la atmosfera. Los vehículos automotores que transitan en los centros urbanos son el mayor contaminador del aire con gases tales como el monóxido de carbono, hidrocarburos y oxidantes fotoquímicos. Los contaminantes provienen de la combustión incompleta de la gasolina de motor en los vehículos que transportan pasajeros dentro los centros urbanos, liberando sustancias al ambiente como aldehídos, monóxido de carbono, óxidos de nitrógeno, hidrocarburos, óxidos de azufre y material particulado.

A falta de redes de monitoreo de calidad del aire en ciudades pequeñas, un método útil y económico para evaluar la calidad es a través de bioindicadores, como los líquenes, definidos por el Dictionary of the Fungi (2008), como una asociación simbiótica autosuficiente de un hongo (micobionte) y un alga (fotobionte). Como no tienen raíces, hojas o flores, toman el agua y sus nutrientes a partir de la atmósfera. Los líquenes epífitos son los que colonizan los troncos y ramas de los árboles y arbustos. Su valor como bioindicadores de la calidad del aire y su sensibilidad frente a cambios ambientales ha sido corroborado por múltiples estudios en el mundo y los hace ser muy utilizados en programas de seguimiento de la contaminación atmosférica, pues cuando los niveles de contaminantes se incrementan estos tienden a desaparecer sobreviviendo solo los más resistentes.

Quibdó, la capital del departamento del Chocó, cuenta con una zona urbana más representativa que en otros municipios y un parque automotor en constante crecimiento, por lo que es importante determinar cuál es la calidad del aire que respiramos y como se diversifica en las diferentes zonas del casco urbano, para que con base en esta información se tomen medidas preventivas y/o correctivas según sea el caso.

De este estudio se obtuvo información detallada de la diversidad y abundancia de especies indicadoras de pureza atmosférica y con base en estos datos se establecieron las zonas de contaminación atmosférica, y se resuelven los siguientes interrogantes: ¿Cuál es la pureza atmosférica en diversos lugares del casco urbano de Quibdó? ¿Cuántas zonas de isocontaminación encontramos en la ciudad y donde están ubicadas?

Área de estudio. La ciudad de Quibdó es la capital del departamento del Chocó, Colombia, se encuentra a orillas del río Atrato, uno de los principales afluentes del país y una de las zonas con más alta pluviosidad del mundo. Está ubicado en la región de las calmas ecuatoriales y según el sistema de Holdrige (1967), corresponde a las zonas de vida de bosque muy húmedo tropical (bmh-T) y bosque pluvial tropical (pb-T), caracterizadas por altas precipitaciones y temperaturas superiores a $24^{\circ} \mathrm{C}$ (Alcaldía de Quibdó, 2001). Tiene un área de $3337.5 \mathrm{~km}^{2}$ y una población de 97.714 habitantes, la cual representa $32 \%$ del total del departamento (DANE, 2005). El 65\% de la población se encuentran en el área urbana. La cabecera municipal Quibdó, se encuentra a $5^{\circ} 41^{\prime} 13^{\prime \prime}$ de latitud norte y $76^{\circ} 39^{\prime} 40^{\prime \prime}$ de longitud este, respecto al meridiano de Greenwich; se encuentra entre 43 y $53 \mathrm{msnm}$ y tiene una temperatura promedio de $28^{\circ} \mathrm{C}$.

Quibdó presenta tres unidades climáticas: cálido super húmedo (Cs), con una extensión aproximada de 275.000 ha, equivalentes a $82.4 \%$ del territorio, en donde se localizan todos los centros poblados del municipio. Medio super húmedo (Mh), con 47.500 ha y $14.2 \%$, se encuentra en esta zona el sector occidental del resguardo de Bebaramá. Muy frío y frío húmedo y perhúmedo (Fh), 11.250 ha, equivalentes al $3.4 \%$, en este sector no se encuentran poblaciones (Alcaldía de Quibdó, 2001).

El casco urbano es un área en constante expansión debido a los procesos de crecimiento de la población y el desplazamiento forzado. Las vías de transporte se encuentran pavimentadas en un $45 \%$, siendo las vías destapadas una fuente de material particulado importante en días soleados y períodos de verano. Como fuentes fijas de contaminación encontramos en la zona algunas chimeneas de establecimientos públicos y privados como restaurantes y asaderos de pollos, 
sitios de ahumado de alimentos, fábricas de materiales de construcción prefabricados, puertos areneros y fluviales, ladrilleras, aserríos, terminal de transporte terrestre, pero la principal fuente de contaminación son las emisiones de gases del parque automotor en constante crecimiento y compuesto por busetas, buses, camionetas, motocicletas y otros.

\section{Métodos}

El muestreo se realizó siguiendo los protocolos establecidos por Kricke y Loppi (2002) para bioindicadores y la metodología de muestreo de líquenes propuesta por Aguirre (1995), usando los formatos de muestreo diseñado por Rubiano (1984).

Establecimiento de estaciones. Para la ubicación de las estaciones de muestreo se dividió el mapa del casco urbano de Quibdó en cuadrículas de $0,5 \mathrm{~km} \times 0,5 \mathrm{~km}$, que incluye las comunas o barrios presentes en el perímetro urbano, resultando 32 cuadrículas, luego se realizó un recorrido preliminar para determinar los siguientes aspectos:

- Abundancia o presencia de forófitos (árboles portadores de líquenes).

Condiciones topográficas.

Árboles sobre las vías o cercanos a estas.

- Cubrimiento de la mayor área posible del casco urbano.

Además, se identificaron como fuente principal de contaminación las emisiones por fuentes móviles del parque automotor de Quibdó y algunas fuentes puntuales de menor afectación como:

. Ladrilleras (barrio Niño Jesús, barrio Jardín, zona minera, vía a Cabí).

- Asaderos y restaurantes con chimeneas (presentes en casi todos los barrios).

Sitios de ahumado de alimentos (La Victoria, cra $7^{\text {a }}$, Niño

Jesús, La Yesquita, Huapango).

- Vías principales y secundarias pavimentadas y destapadas.

- Fábricas de materiales de construcción prefabricados (calle 29, vía a Cabí).

Puertos areneros y fluviales.

Terminal de transporte terrestre (vía a Cabí).

Aserríos (Playita, carrera $1^{\text {a }}$, calle 20 , entre otros).

De las 32 cuadrículas iniciales se eliminaron aquellas que no cumplían con las condiciones requeridas como presencia de forófitos, forófitos con diámetro mayor a $20 \mathrm{~cm}$, fácil acceso, etc., quedando 21 estaciones apropiadas para realizar el muestreo (Figura 1):

2. Barrio La Unión-La Victoria.

3. Barrio La Fe-Obrero.

7. Barrio Huapango- Subestación después de la Cra $10^{\mathrm{a}}$.

8. Margen derecha del Río Atrato: Barrio Kennedy, desde la Obapo ONG hasta la calle 31.
9. Cra 6 hacia Tomas Pérez, Subestación hasta calle 31.

10. Club Telecom, barrio Porvenir.

11. Barrio Caraño, vía a Medellín.

12. Cra $1^{\text {a }}$ hasta cra $5^{\text {a }}$. Calle 31 hasta calle 27 .

13. Cra 5a hasta cra 12. Barrio San Judas parte baja-Silencio.

14. Barrio Santa Ana parte baja y barrio Las Américas.

15. Aeropuerto y alrededores.

16. Calle 26 hasta calle 20 . Cra $1^{\text {a }}$ hasta cra $6^{\mathrm{a}}$.

17. Cra $7^{\mathrm{a}}$ hasta cra 23. Calle 27 hasta calle 20 .

22. Acueducto.

24. Minercol.

27. Barrio Castillos-Batallón.

30. Barrio Horizonte.

31. Los Rosales.

32. Vía Cabí.

Selección de forófitos. En cada una de las estaciones seleccionadas se escogieron 4 árboles teniendo en cuenta los siguientes requerimientos:

Abundancia o presencia de la especie del forófitos (árboles portadores de líquenes) hasta donde fue posible, las mismas especies en cada estación.

Facilidades de acceso.

- Árboles expuestos, sin interferencias de casas, plantaciones o accidentes del terreno, ni interferencias antropogénicas.

- Sin daños fitosanitarios o antropogénicas o de animales. - Diámetro del árbol superior a $20 \mathrm{~cm}$ y fuste vertical.

Se muestrearon en total 84 árboles, tratando de utilizar en lo posible las mismas especies en cada estación censada, no obstante la comunidad arbórea en el casco urbano de Quibdó se caracteriza por su diversidad, por lo tanto en algunas estaciones se tomaron especies diferentes (Figura 2).

El muestreo se realizó en la cobertura del árbol entre 0 y 2 metros de altura y alrededor de la circunferencia (Figura 3). Además de los líquenes se muestrearon las especies de briofitas acompañantes, pues representan biodiversidad y también son bioindicadoras (Bedregal et al. 2005, Monnet et al. 2005). La abundancia se cuantificó midiendo la cobertura en el árbol, mediante una plantilla transparente con cuadriculas de $1 \mathrm{~cm}^{2}$. Asimismo se determinó su vitalidad, teniendo en cuenta su apariencia y su nivel de necrosamiento o muerte; utilizando una escala de 1 a 4 donde 1 equivale a excelente apariencia y 4 a necrosamiento total del líquen. A los forófitos se les georeferenció con un GPS, se midió su diámetro, inclinación y la altura del fuste. Se reporta el nombre común y científico.

De cada especímen detectado se tomaron muestras, que fueron clasificadas y registradas en el catálogo. Luego las muestras fueron secadas al sol por 48 horas.

La identificación taxonómica se realizó utilizando claves dicotómicas por especialistas en plantas no vasculares, los 


\section{Bioetnia Volumen 9 № 2 (julio-diciembre), 2012}

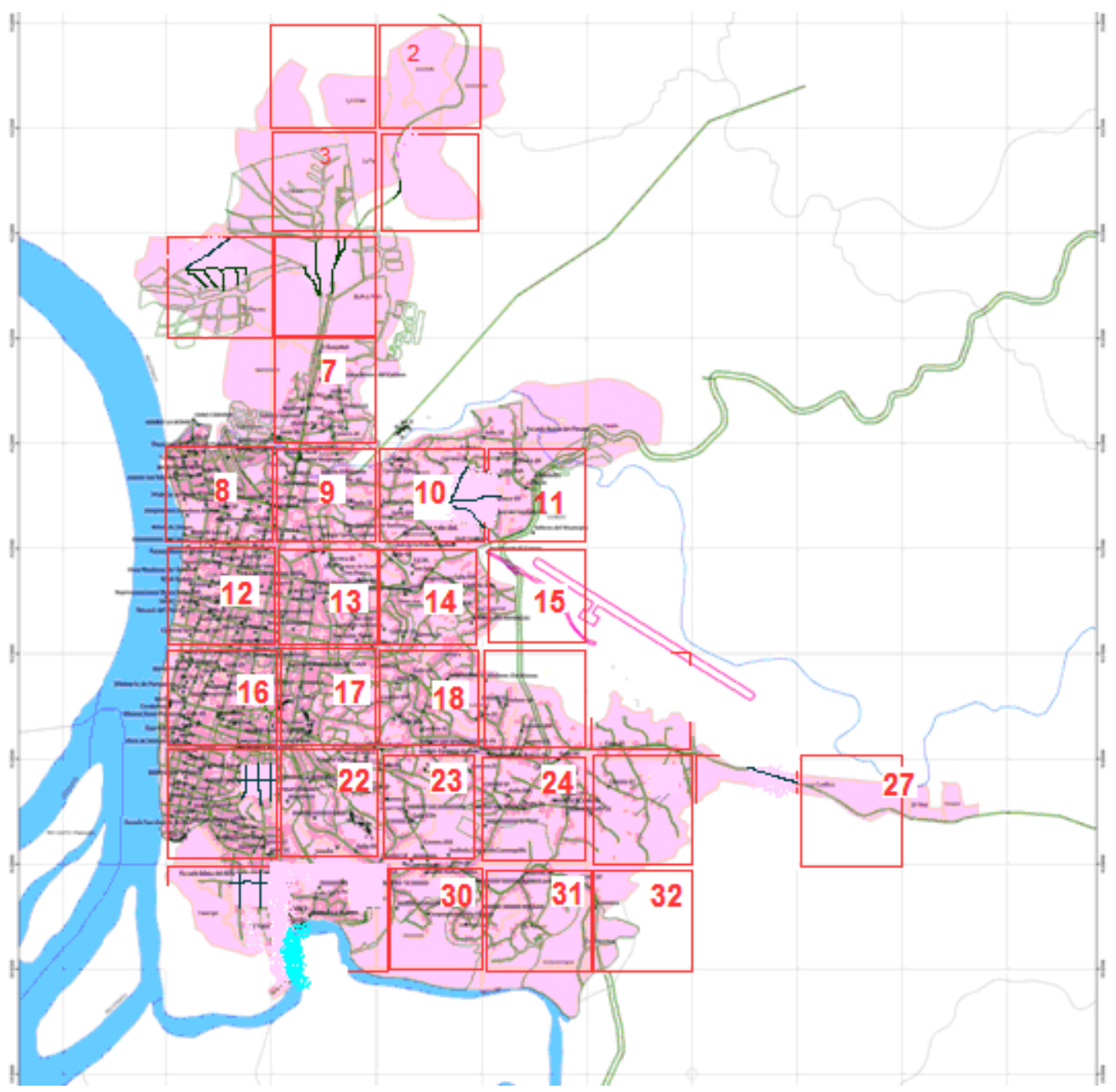

Figura 1. Estación de muestreo

especímenes que no pudieron ser identificados por encontrarse estériles, fueron enviados a análisis más avanzados (cromatografías) para determinar su género y especie hasta donde fue posible.

Determinación del Índice de Pureza Atmosférica (IPA). El IPA (Le Blanc y De Sloover, 1970) relaciona en una sola expresión cobertura, diversidad y frecuencia de especies de líquenes. La fórmula original fue modificada por Rubiano (1984), incorporando el criterio de coberturas relativas. La fórmula es la siguiente:

$$
\text { IPAj = Ei Qi.fi. Cri/n }
$$

IPAj = IPA de la estación j

$\mathrm{fi}=\quad$ Frecuencia de la especie i (número de forófitos de la estación j en que aparece la especie)

$\mathrm{Cri}=$ Cobertura relativa de la especie $\mathrm{i}$ en la estación j respecto a la cobertura máxima de la especie i en el área estudiada. La cobertura para cada estación es la suma de la cobertura en cada forófito.

$n=\quad$ Número de forófitos censados en la estación j

$\mathrm{Qi}=$ Factor de resistencia definido como el número promedio de especies acompañantes de la especie i en todas las estaciones y se deduce mediante el cociente

$Q i=\varepsilon j(A j-1 / E j)$ donde:

$\mathrm{Qi}=$ Factor de resistencia de la especie $\mathrm{i}$

$\mathrm{Aj}=\quad$ Número de especies presentes en cada estación donde se encuentre i

$E j=$ Número de estaciones donde se encuentra $\mathrm{i}$

El IPA tiene un valor máximo teórico igual a $\mathrm{S}^{2}$-S donde $\mathrm{S}$ es el número total de especies encontradas en la zona de estudio y solo tiene validez local pues está definido por la riqueza de especies liquénicas de cada zona.

La determinación de áreas homogéneas en cuanto al 


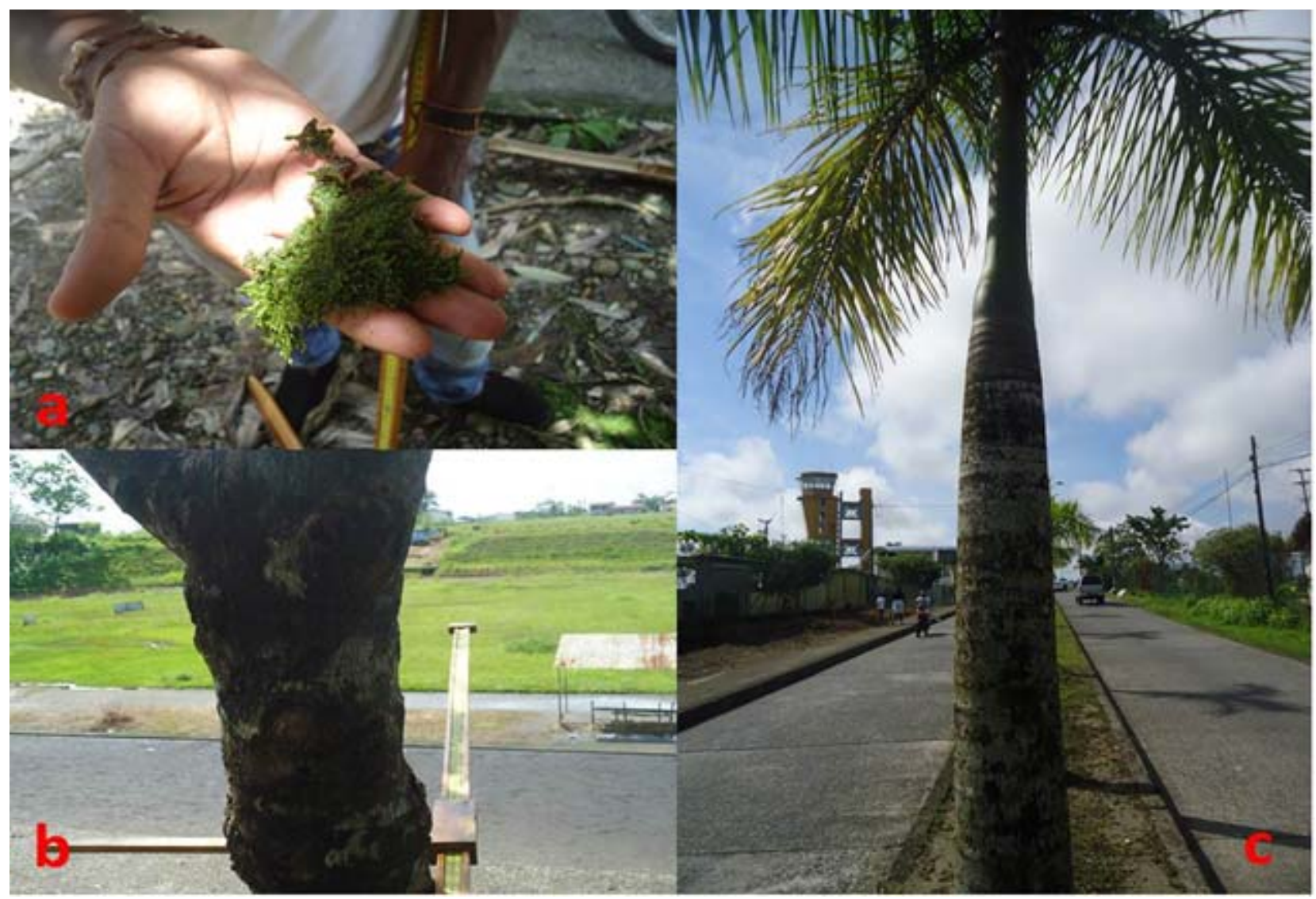

Figura 2. a. Toma de muestras de briofitas. b. Medición de diámetro de forófitos con forcípula. c. Líquenes en palma Estación Aeropuerto

comportamiento de las comunidades liquénicas corresponde a áreas similares por niveles de contaminación (Rubiano, 1989). Con el objeto de definir estas áreas se agruparon los IPA registrados, en clases de forma arbitraria de acuerdo con la dispersión y/o la similitud de los datos.

Para delimitar gráficamente las isolíneas en el mapa, se marcaron los puntos correspondientes a las estaciones de muestreo y se unieron de acuerdo con los rangos de valores y zonas establecidas mediante el programa ArcGis para sistemas de información geográfica.

\section{Resultados y discusión}

Se muestrearon en total 84 árboles de los cuales $69,1 \%$ de las especies corresponde a cuatro grupos más frecuentes en zonas urbanas y $30,9 \%$ restante pertenece a diferentes especies encontradas. Las especies más frecuentes fueron palmas: palma real (Roystonea regia), palma de coco (Cocos nucifera), ficus (Ficus sp.), marañon (Eugenia malasensis) y guamo (Inga sp.). En el porcentaje restante se identificaron las especies de roble (Taebuia rosea), pino (Araucaria columnaris), ilan ilan (Cananga odorata), acacia (Caesal- pinia peltophoroides) y los frutales de mango (Mangifera indica L) y árbol del pan (Artocarpus altilis), coronilla (Bellucia pentamera), almendro (Terminalia catappa). Las especies presentes en las vías principales y secundarias descritas antes, son especies foráneas que han sido introducidas en la ciudad y hacen parte del ornamento urbano, a excepción de los árboles de coronilla (Bellucia entamera) que son nativas.

Comunidades de líquenes. Los líquenes pueden clasificarse en tres grandes grupos de acuerdo con las características del talo: foliosos, en forma de hojas o láminas; fructicosos, con ramificaciones; y crustáceo o costroso en forma de costra, adheridos a la corteza del árbol. De las 74 especies colectadas se diferenciaron 34 (45,9\%) líquenes costrosos, 9 $(10,8 \%)$ líquenes foliosos y un fructicoso $(1,4 \%)$. Además, se identificaron 13 tipos de musgos equivalentes al 17,6\%; 14 especies de hepáticas pertenecientes a la familia Legeuneaceae, que corresponde al $18,9 \%$ de los especímenes; y se detectó un 5,4\% de micelios de hongos en las muestras.

Estos resultados son concordantes con lo reportado por Aguirre y Sipman (2004), quienes afirman que las formas de crecimiento crustáceas son los más abundantes en el Chocó 


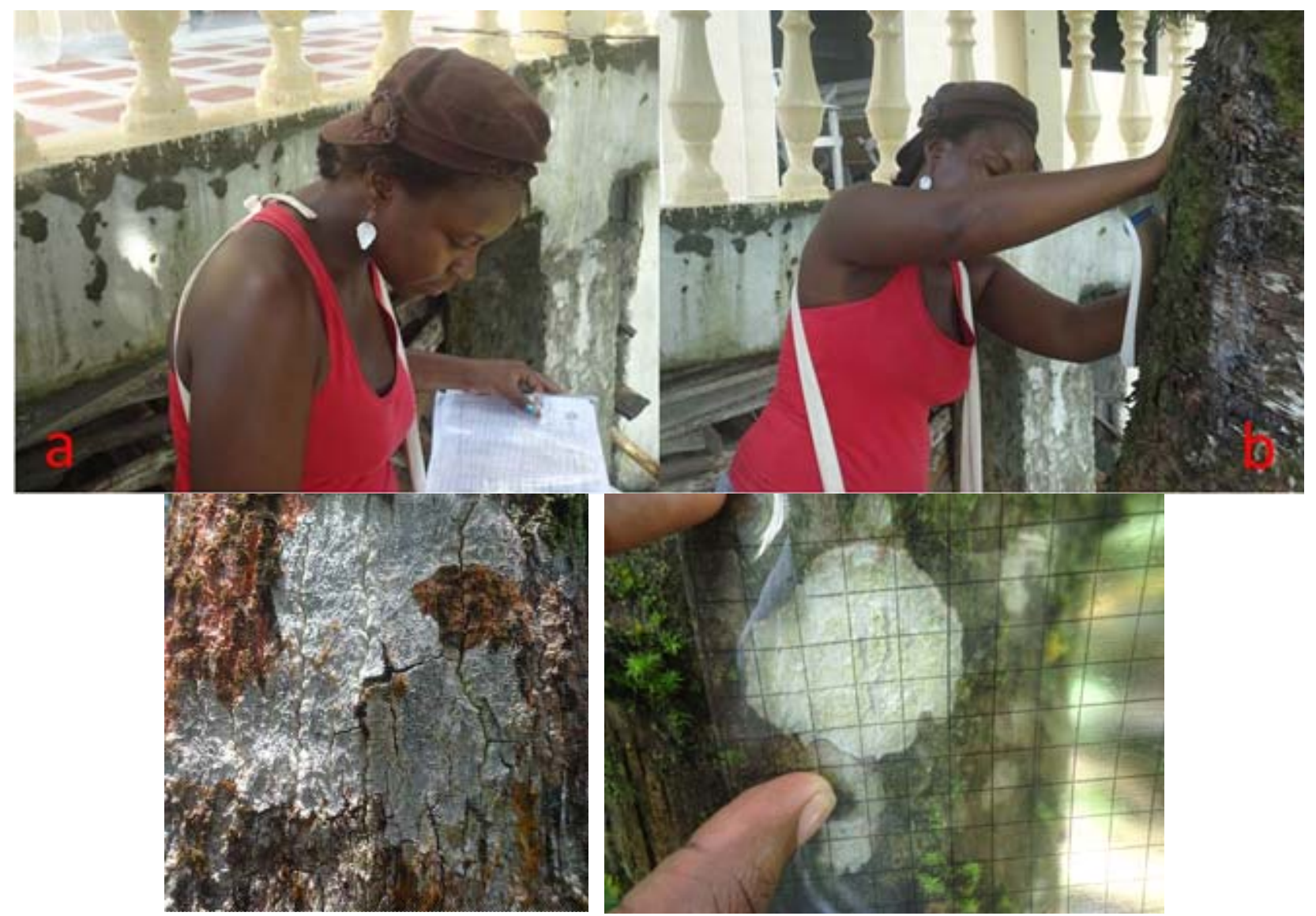

Figura 3. a. Trabajo de campo, captura de la información.

b y d. Medición de la cobertura de epífitos. c. Liquen Graphis sp.

Biogeográfico y que son escasos los líquenes fructicosos. Por su parte Lakatos et al. (2006) atribuye esta abundancia de líquenes costrosos, a las adaptaciones fisiológicas que presentan estos organismos ante las altas concentraciones de humedad y baja intensidad lumínica, propias de los sotobosques, condiciones fácilmente encontradas en la ciudad de Quibdó y cuyos registros mensuales de humedad relativa alcanzan entre $80 \%$ y 90\% según Alcaldía de Quibdó (2000).

En la Tabla 1 se observa el listado de especies encontradas, ordenadas en forma descendente de acuerdo con su resistencia, las zonas de similares características atmosféricas y las estaciones pertenecientes a estas. Las especies más resistentes de acuerdo el factor Qi fueron: Graphis sp. 4, Legeuneaceae sp. 1, Graphis sp. 5, Graphis sp. 3 y Plagiochila sp. 2, presentes en las zonas I y II de contaminación máxima y aguda.

De acuerdo con estos resultados el factor de resistencia Qi de cada especie, diseñado por Le Blanc y De Sloover (1970) y que está definido como el número promedio de especies acompañantes de la especie $i$ en todas las estaciones, sí evidencia altos niveles de contaminación al relacionar la dominancia de unas especies en situaciones adversas frente a las demás; lo que coincide con lo expuesto por Rubiano (1983) que infiere que para una serie de registros hechos bajo condiciones homólogas de muestreo, una especie sería resistente cuando se desarrolla y propaga en condiciones de aire contaminado, situación que coincide generalmente con una reducción en el promedio de sus especies acompañantes.

De las familias identificadas taxonómicamente se encontró una mayor frecuencia y mayores coberturas en cuanto a líquenes, a la familia Graphidaceae, y de briofitas a la familia Legeuneaceae (Figura 4), ambas presentes en casi todas las estaciones independientemente del nivel de contaminación y representadas con 7 y 13 especies, lo cual se debe a sus características cosmopolita que según Aguirre et al. (2011) les permite colonizar diferentes hábitats, incrementado así su frecuencia de registro en un muestreo. Cabe mencionar que un porcentaje de líquenes costrosos se encontraron sin estructuras reproductivas (estériles) dificultando su identificación taxonómica, lo que pudo estar relacionado con lo expuesto por Hawksworth y Rose (1970), quienes indican que este tipo de estructuras no se desarrolla o se deterioran en 


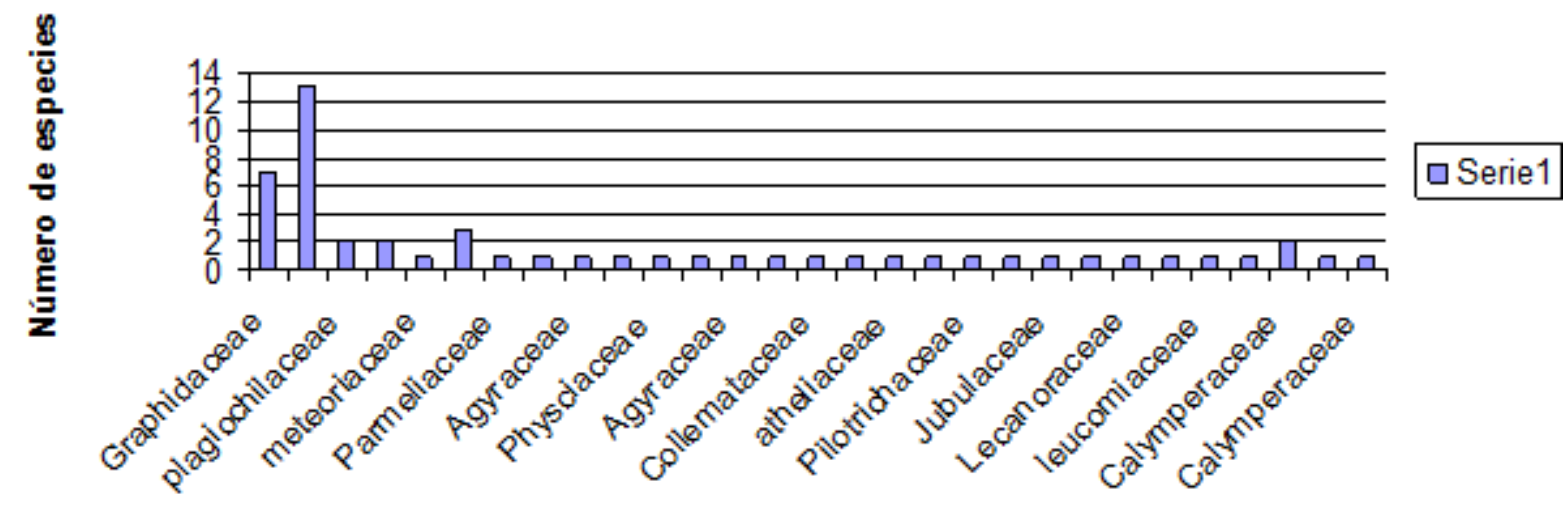

Familias

Figura 4. Frecuencia de familias identificadas

ambientes donde los líquenes están expuestos a contaminación, como los muestreados en el presente estudio.

Los cálculos de los IPA dieron como resultado valores en un rango entre 5,2 y 58,5 y se agruparon en cinco clases teniendo en cuenta las características de las estaciones, diversidad epifita y coberturas. En la Tabla 2 podemos observar las zonas correspondientes y sus niveles cualitativos de contaminación.

El IPA combina en una expresión la cobertura, diversidad y frecuencia de las especies indicadoras en un área determinada (Rubiano, 2006). El IPA máximo teórico se calcula con el número total de especies encontradas en la zona de estudio y según Eljaude y Taborda (1995) solo tiene validez local pues está definido por la riqueza de especies liquénicas de cada zona. El calculado para la zona urbana de Quibdó es igual a:

\section{IPA máx. Teórico $=\mathrm{S}^{2}-\mathrm{S}$}

Donde $\mathrm{S}$ es el número total de especies encontradas en la zona de estudio

$$
\begin{gathered}
\text { IPA máx. teórico (Quibdó) = } \\
74^{2}-74=5402
\end{gathered}
$$

El IPA máximo teórico equivaldría a una situación ideal donde el número total de especies encontradas estuviera presente en todas las zonas con un máximo de cobertura en todos los árboles censados. Es un número de verificación que fija un umbral para el indicador. De acuerdo con los resultados del IPA calculado, se identificaron cinco zonas de características similares de calidad de aire en el área de estudio, las cuales se listan y describen a continuación en la Tabla 3 con sus valores IPA correspondientes.

La Zona I, de contaminación máxima, presenta los valores IPA más bajos observados (entre 5.2 y 9.2) y corresponde a las estaciones 27, 30,12, 15, 10, 9 y 16. (33,3\% del área estudiada). La Estación 27 se encuentra localizada en la vía a Pacurita, cerca al batallón Manosalva Flórez del Ejército y se caracteriza por un alto tráfico vehicular y por estar destapada lo que genera no solo contaminación por emisiones de fuentes móviles, sino también una gran producción de material particulado proveniente de la superficie durante el flujo vehicular, lo que justifica el resultado obtenido y su inclusión dentro de la zona I. Para el caso de la Estación 30, ubicada en la congregación religiosa Mita en Aaron, aunque el flujo vehicular es escaso, cuenta con una fábrica de alimentos horneados al carbón, la cual podría ser una fuente importante de $\mathrm{CO}$ y $\mathrm{CO}$, hollín y otros gases a altas temperaturas, que gracias a las condiciones de la edificación permiten que dichos gases se concentren en el área, afectando así la población de epífitos.

Las estaciones $12,9,10$ y 16 corresponden a la parte céntrica del casco urbano, caracterizada por ser el centro comercial de la ciudad, donde se encuentran la mayor parte de entidades (públicas y privadas) y viviendas, de ahí que cuente con mayor tráfico vehicular. En estas estaciones está inmerso el anillo asfáltico, principal zona de circulación de automotores de servicio público. La Estación 15, corresponde a la vía conducente al aeropuerto, la cual presenta un alto tráfico vehicular y conecta varios núcleos residenciales de la ciudad, que explica los bajos índices de pureza obtenidos en esta área. Los epífitos en esta zona se caracterizan por tener algún grado de necrosamiento, pérdida de color y disminución de coberturas de algunas especies. Según Crespo et al. (1977), esta alteración se presenta en ambientes contaminados, posiblemente como consecuencia de la pérdida de iones que induce alteraciones en las membranas celulares del alga asociada y ruptura de los mecanismos homeostáticos.

La Zona II, de contaminación aguda, corresponde a $42,9 \%$ del área de estudio y comprende las estaciones 24,8 , $11,23,22,31,32,13$, y 7 donde se muestrearon forófitos en 
Bioetnia Volumen 9 № 2 (julio-diciembre), 2012

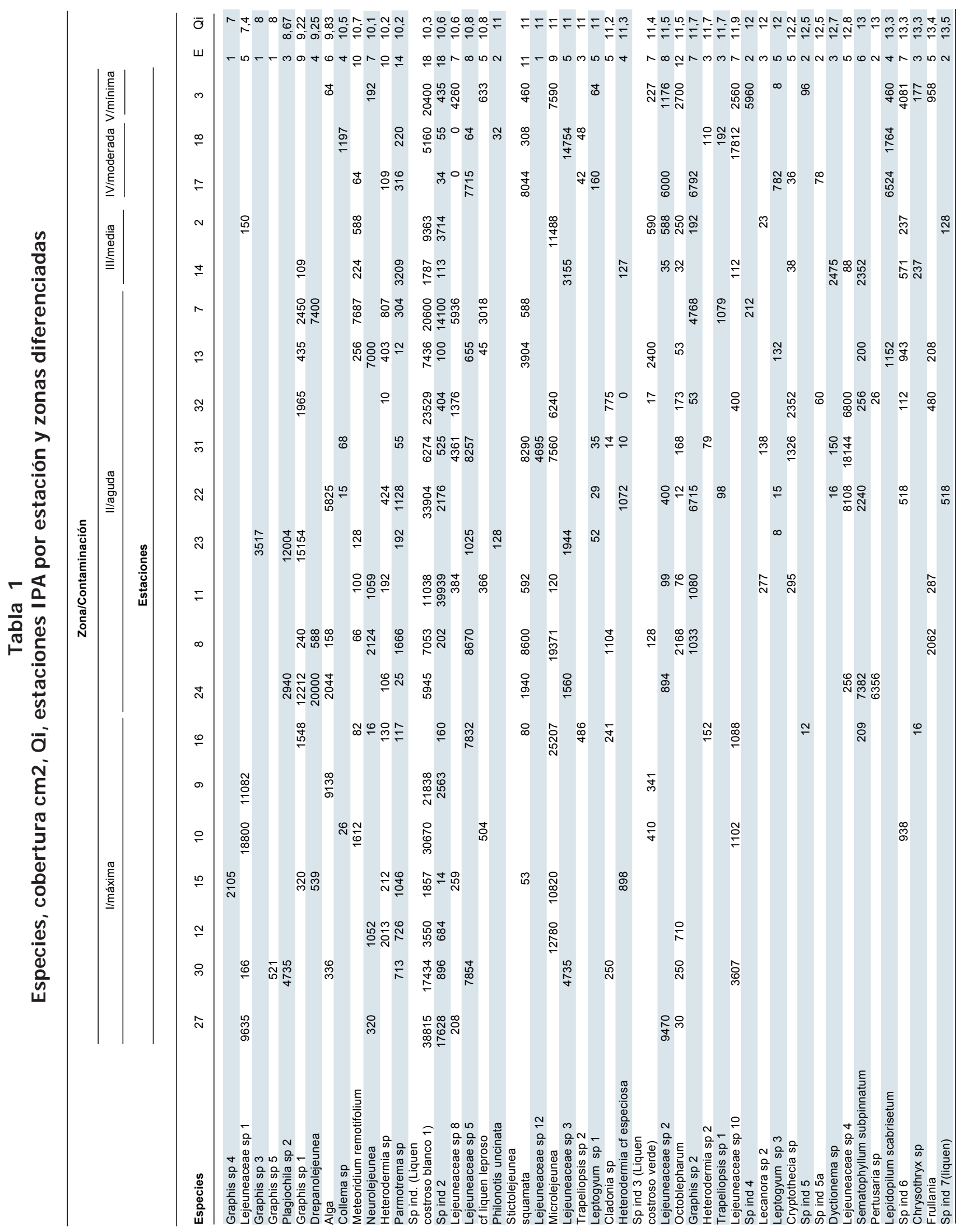




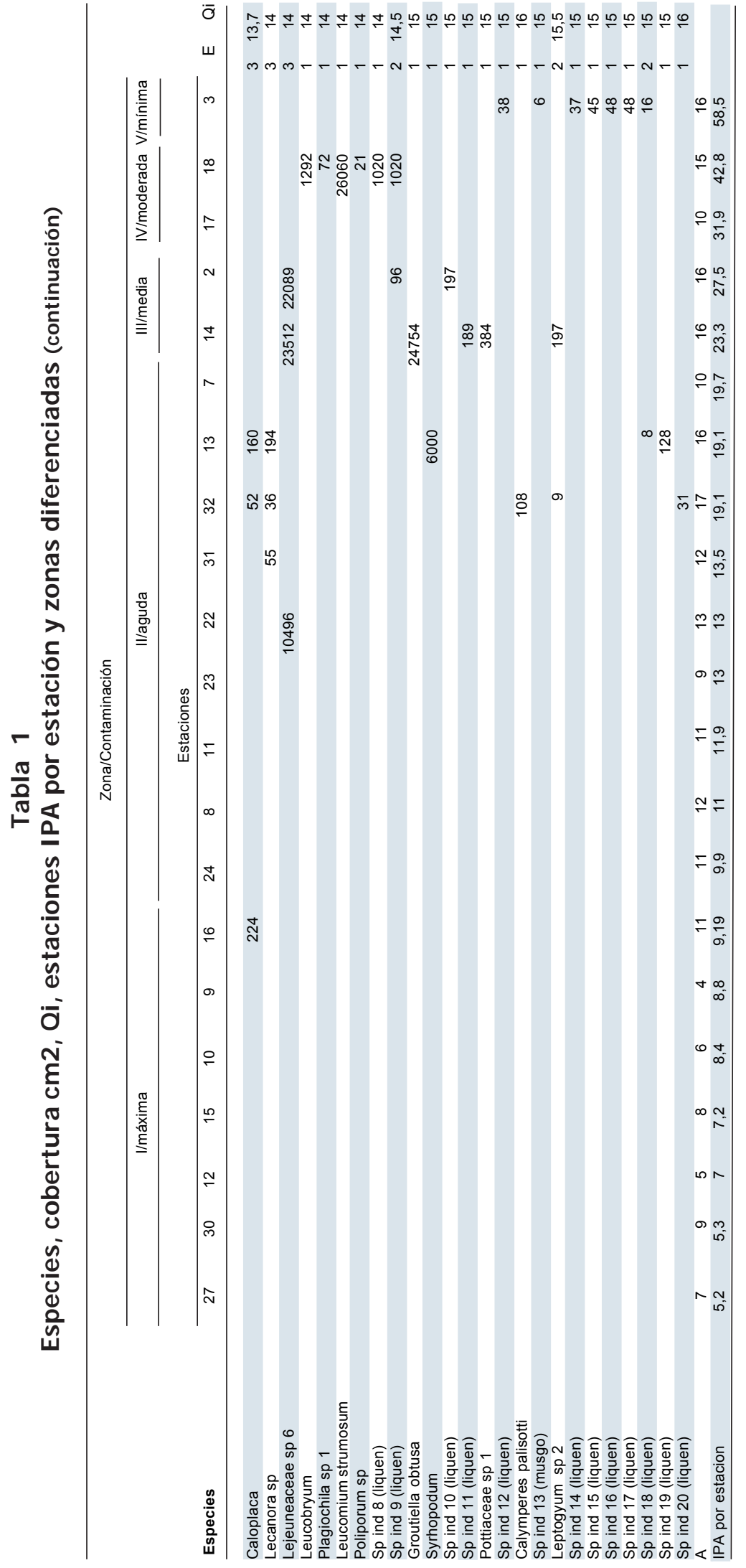

Minercol, IFEMP, Caraño, Ancianato, Acueducto, UTCH, Cabí, Carrasquilla, y Casa de Justicia respectivamente. Los valores IPA de esta zona están dentro del rango de 10.1 a 20 en la cual se colectaron 12 especies. De esta hacen parte los barrios de Kennedy, Huapango, Silencio, El Caraño, Bonanza, Piñal, Jardín, Niño Jesús, vía a Cabí. Este sector es en su mayoría residencial, caracterizado por vías muy transitadas. De ahí que los valores IPA encontrados en estas estaciones podrían estar relacionados con el patrón de crecimiento de la ciudad, caracterizado por calles angostas y sin ordenamiento claro, vías destapadas y la escasez de zonas verdes o arborizaciones las cuales han sido desplazadas por las obras civiles. En barrios como el Jardín, Kennedy, Silencio, Niño Jesús, Bonanza y el Piñal se encontraron pocos forófitos, los cuales han sido sembrados en algunas instituciones como el Colegio Carrasquilla y la EPM.

En términos generales las zonas Iy II presentaron mayor índice de contaminación, debido a que corresponden al centro de la ciudad donde confluyen residencias, comercio, instituciones, parques recreativos, paraderos de buses, parqueaderos, supermercados, oficinas, almacenes, ventas ambulantes e instituciones educativas; su denso nivel de ocupación del suelo por diferentes actividades de tipo antrópico hace que haya una mayor liberación de sustancias contaminantes a la atmósfera lo que está directamente relacionado con el comportamiento de la calidad liquénica y su bioindicación de la calidad del aire.

La Zona III, contaminación media pertenece a las estaciones 14 y 2 ubicadas en la Victoria y el Cementerio. El muestreo se realizó en la vía principal que conduce a la zona norte de la ciudad y en la Estación 14 en el cementerio y el edificio de ISS o Caprecom. Corresponde al 9,5\% de las estaciones muestreadas. Los valo- 
Bioetnia Volumen 9 № 2 (julio-diciembre), 2012

Tabla 2

Rangos I PA

\begin{tabular}{lll}
\hline Clase & \multicolumn{1}{c}{ Zona } & \multicolumn{1}{c}{ Rango I PA } \\
\hline I & Contaminación máxima & IPA mínimo a 9.5 \\
II & Contaminación aguda & 9.5 a 20 \\
III & Contaminación media & 20,1 a 30 \\
IV & Contaminación moderada & 30,1 a 50 \\
V & Contaminación mínima & 50,1 a IPA máx. \\
\hline
\end{tabular}

Tabla 3

Zonas de isocontaminación

\begin{tabular}{|c|c|c|c|}
\hline Zona & Clase & Estación & Valor I PA \\
\hline \multirow{7}{*}{ Zona I } & \multirow{7}{*}{ Contaminación maxima } & 27. Herradura & 5,2 \\
\hline & & 30. Mita & 5,3 \\
\hline & & 12. Sena-Malecon & 7 \\
\hline & & 15. Aeropuerto & 7,2 \\
\hline & & 10. Sto Domingo & 8,4 \\
\hline & & 9. Cra 6ta & 8,8 \\
\hline & & 16. Fiscalía & 9,2 \\
\hline \multirow{9}{*}{ Zona II } & \multirow{9}{*}{ Contaminación aguda } & 24. Minercol & 9,9 \\
\hline & & 8. IFI & 10,9 \\
\hline & & 11. Caraño & 11,9 \\
\hline & & 31. UTCH & 13 \\
\hline & & 23. Ancianato & 13 \\
\hline & & 22. Acueducto & 13,5 \\
\hline & & 13. Carrasquilla & 19,1 \\
\hline & & 32. Cabí & 19,1 \\
\hline & & 7. Casa Justicia & 19,7 \\
\hline \multicolumn{4}{|c|}{ Zona III Contaminación media } \\
\hline \multirow{2}{*}{ Zona IV } & \multirow{2}{*}{ Contaminación moderada } & 17. Cohimbra & 31,9 \\
\hline & & 18. Margaritas & 42,8 \\
\hline Zona V & Contaminación mínima & 3. Cachamas & 58,5 \\
\hline
\end{tabular}

res IPA obtenidos fueron 23,3 y 27,5 respectivamente. En esta área se colectaron 16 especies en cada estación y se observa un incremento en el IPA debido a que disminuye el tráfico vehicular y con ella las emisiones contaminantes.

La Zona IV, contaminación moderada, cuenta con 2 estaciones (17 y 18), corresponde al área que coincide con los barrios Margaritas, parte del Jardín, Minuto de Dios, Esmeralda, Cohimbra, Santa Ana y Loma de San Judas, los cuales constituyen zonas residenciales, que conservan porciones de áreas verdes, una menor contaminación y menor afluencia de vehículos.
La Zona $\mathrm{V}$, contaminación mínima, corresponde a la Estación 3 que presentó mayor riqueza de especies y mayor cobertura de las mismas. Se obtuvo el IPA máximo de todas las estaciones: 58,5. El muestreo se realizó cerca al Parque Natural Las Cachamas, ubicado en la zona de expansión en la parte norte de Quibdó, caracterizada por su ocupación residencial, hasta el momento con una baja densidad y zonas con poca intervención.

Las zonas III, IV y V, de contaminación media, moderada y mínima corresponden a zonas más altas de la ciudad, con altitudes superiores a $50 \mathrm{msnm}$. En estas áreas se encontraron especies exclusivas como plagiochila, leucobryum y líquenes costrosos. La ubicación de las zonas y su distribución aparecen en la Figura 5.

Los resultados obtenidos y el patrón de comportamiento de los líquenes permitió evaluar la calidad del aire en el casco urbano de Quibdó, en las diferentes zonas, siendo el incremento del parque automotor y el tráfico vehicular en sus vías la fuente principal de contaminación; en este caso hay una relación con la disminución de la cobertura, diversidad y vitalidad de epifitos; de ahí que la zona más contaminada se encuentre ubicada en la zona céntrica de la ciudad y barrios cercanos a vías principales.

En sitios como Argentina se estableció la correlación entre la supervivencia de ejemplares transplantados de la especie Ramalina ecklonii, con la densidad del tráfico automotor (Leving, 1995), corroborando el impacto de las emisiones de fuentes móviles en las comunidades liquénicas. Existen otros estudios que evidencian que estos contaminantes vehiculares afectan la vegetación liquenológica como Lijteroff et al. 2009, Fuga et al. 2008, Anze et al. 2007, Giordani 2007, Jovan 2007, Thormann 2006, Weissman et al. 2006, Monge-Nájera et al. 2002a, Conti y Cecchetti2001, Garty et al. 2000, Vokou et al. 1999, quienes han informado esta 
Calidad del aire en Quibdó mediante bioindicadores. R. Mena

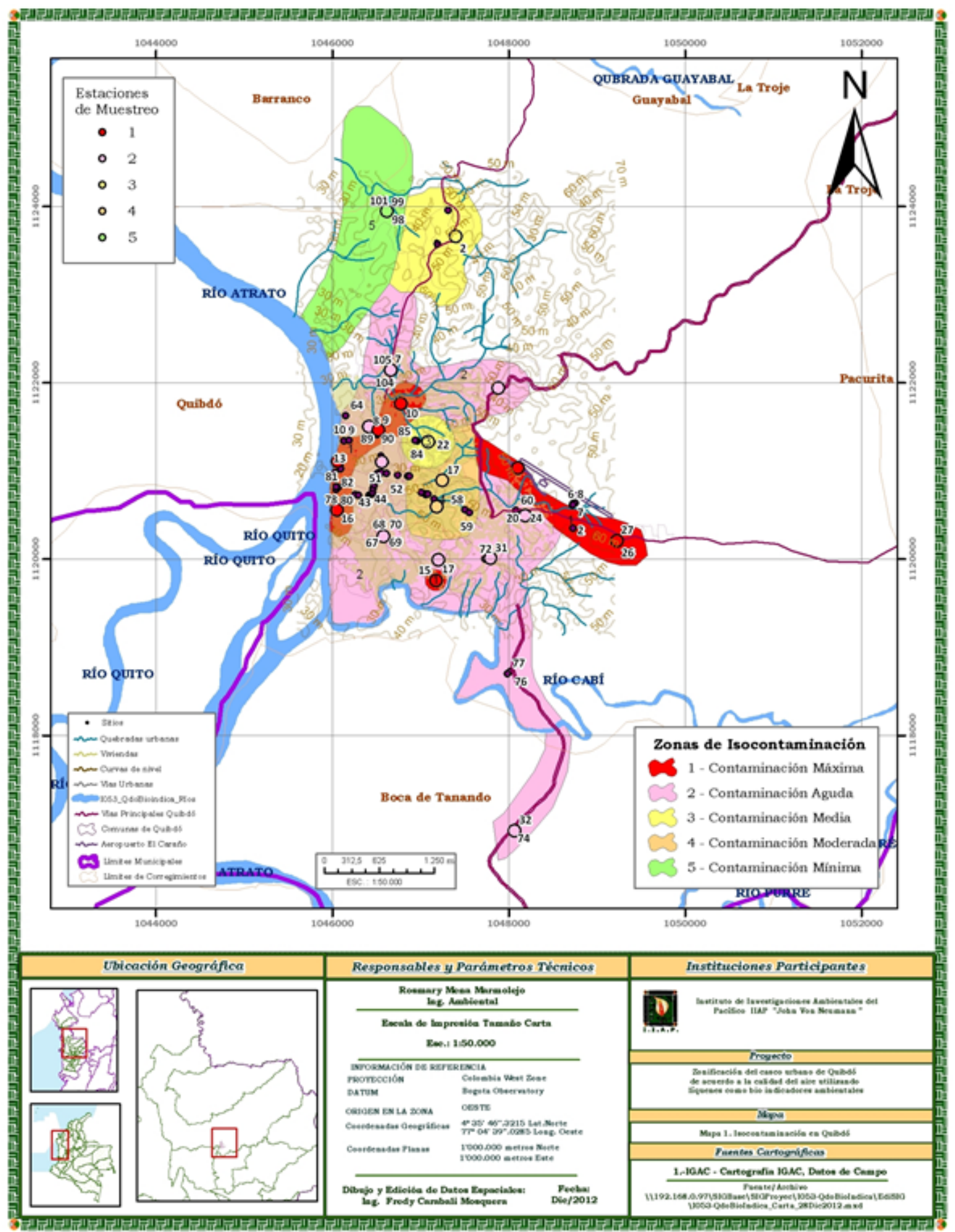

Figura 5. Zonificación de Quibdó de acuerdo con calidad del aire 


\section{Bioetnia Volumen 9 № 2 (julio-diciembre), 2012}

relación mediante análisis y muestreos realizados en diferentes sitios del mundo.

El patrón de crecimiento urbano en la ciudad ha dejado de lado la vegetación primaria para ser reemplazada por construcciones, sin tener en cuenta en casi todas las comunas espacios de esparcimiento arborizados o zonas verdes, los cuales servirían como barreras naturales contra la contaminación atmosférica. La presencia de especies ornamentales en las vías debería ser una constante para mejorar la calidad del aire. No obstante para el casco urbano de Quibdó, la diversidad encontrada es alta si se compara con otras ciudades del país donde se han realizado estudios similares; sin embargo existe una clara diferenciación en las zonas de mayor tránsito vehicular, donde se obtuvieron los valores de IPA más bajos. Por ejemplo Eljaude y Tabora (1995) encontraron para Bogotá un valor de 1,7 como el mayor IPA con 5 especies de líquenes colectadas, el IPA máximo teórico fue de 20. Por otro lado, Rubiano (1986) encontró índices que variaron de 0 a 67,15 en Medellín y 32 especies; de 0 a 115,8 en Cali, con 35 especies. Ambas ciudades diferenciadas en cinco zonas de isocontaminación. En las ciudades mencionadas antes se encontraron desiertos de líquenes en las zonas más contaminadas y se les asignó un valor IPA igual a 0 ; en el caso Quibdó no existen zonas sin líquenes, el IPA más bajo fue de 5,2 con 7 especies.

El uso de bioindicadores para detectar la polución tiene una serie de ventajas tales como ser un método más rápido y económico comparado con otras técnicas, y el IPA resulta ser útil para describir la relación entre la disminución de especies y el aumento de contaminantes. Tiene como desventajas que requiere la homologación de las estaciones, características de los forófitos y condiciones que se dificultan para ciertas zonas. García y Rubiano (1984) mencionan que la viabilidad de los líquenes como bioindicadores requiere que los sitios de muestreo sean, en cuanto a su hábitat, lo más uniforme posible con el objeto de que la varianza poblacional y de la comunidad sea mínima para factores diferentes de calidad del aire.

Para este estudio la homologación de forófitos no fue posible por las condiciones particulares del sitio; sin embargo se encontró abundante cobertura de epifitos en los diferentes taxones muestreados, lo que concuerda con los resultados de los estudios que se han realizado acerca de los rangos y patrones de preferencia de los líquenes foliáceos en relación con su forófitos (hospedante), que fueron estudiados por Lücking (1998) en una selva nublada de Costa Rica, el cual descubrió que en áreas de alta diversidad, particularmente en bosques tropicales lluviosos de tierras bajas, las preferencias de un líquen por un forófitos específico son bajas y cuantitativas más que cualitativas y se ve afectado realmente por las condiciones medioambientales, particularmente debido a la intensidad de la luz (Hawksworth et al., 2005). Sería interesante establecer relaciones entre líquenes cortícolas y diferentes hospederos en el bosque húmedo tropical.

En términos generales cualquier cambio desfavorable identificado de los patrones de desarrollo y diversidad de las especies epífitas, debe constituirse en una alarma y propiciar la realización de estudios especializados y la implementación de medidas preventivas y correctivas antes de que los efectos de los contaminantes sobre las comunidades sean aún peores. Los niveles preocupantes de contaminación se presentaron en zonas densamente pobladas del casco urbano, con una tendencia a aumentar por falta de estrategias. Es importante el establecimiento de políticas ambientales territoriales tendientes a mejorar la calidad del aire en Quibdó, que pueden propender por el desarrollo de programas y proyectos para el control de la contaminación como:

- Formulación de mecanismos técnicos para la minimización de emisiones atmosféricas automotoras.

- Optimización de sistemas de transporte y actualización del parque automotor.

- Diseños de mecanismos económicos para el control de la contaminación.

- Establecimiento de una red de vigilancia de calidad del aire utilizando bioindicadores.

- La arborización en las vías y establecimiento de zonas verdes.

- Revisión del Plan de Ordenamiento Territorial en cuanto a uso y ocupación del suelo urbano.

\section{Conclusiones}

El uso de bioindicadores y el cálculo del IPA, dio como resultado que la calidad del aire en el casco urbano de Quibdó, presenta una clara diferenciación que varió de las zonas con mayor tráfico vehicular y mayor densidad en el uso del suelo, a las zonas más apartadas del centro de la ciudad y de las vías principales, mostrando disminución en el número de especies epifitas y su cobertura.

El uso del IPA es un método útil y efectivo para relacionar la presencia y características de los bioindicadores de la calidad del aire y los contaminantes atmosféricos; así como facilita el establecimiento de los rangos que permiten comparar unas zonas con otras dentro de un área homologa de estudio; más no con los valores obtenidos en otros lugares pues no tendría equivalencias con las obtenidas en otras ciudades, ya que la división de las clases depende del criterio del investigador. Para la realización de futuros estudios relacionados se recomienda revisar la metodología desarrollada en este trabajo en busca de optimizar y disminuir el tiempo de muestreo y la extensión de los censos de las comunidades con el objetivo de establecer mayor eficiencia. 


\section{Agradecimientos}

Al Instituto de Investigaciones Ambientales del Pacífico (IIAP) por la financiación, en especial a su director William Klinger Braham por creer en el proyecto y a la ingeniera Lady Vargas Porras, por los aportes en el desarrollo del trabajo. Al biólogo Jair Nagles de la Universidad Tecnológica del Chocó, por su apoyo en el trabajo de campo. Al profesor Edgar Leonardo Linares C. de la Universidad Nacional de Colombia por la identificación taxonómica de briofitas y a Luis Rubiano de la Universidad Nacional por la asesoría.

\section{Literatura citada}

Aguirre, J., M. Ch. de Valencia. 1995. Líquenes, morfología, anatomía y sistemática. Bogotá: Centro de Publicaciones de Física. Universidad Nacional de Colombia. 142 pp.

Alcaldía Municipal de Quibdó. 2001. Plan de Ordenamiento Territorial. Diagnóstico territorial. 1-489 pp.

Bernasconi, E. (1999). Estudio estacional comparativo del Índice de Contaminación (IC) en la ciudad de San Luis utilizando al líquen (Tayl.) como bioindicador. Tesis de Licenciatura. San Luis: Universidad Nacional De San Luis.

Calatayud-Lorente, V., Sanz Sánchez, M. 2000. Guía de líquenes epífitos. Madrid: Organismo Autónomo Parques Nacionales. 185 pp.

Eljadue, L. 1995. Determinación de áreas de isocontaminación en la zona urbana de Santa Fe de Bogotá DC mediante la utilización de líquenes. Trabajo de grado (Lic. Química y Biología). Bogotá: Universidad de la Salle. Facultad de Ciencias de la Educación. Departamento de Química y Biología.

Fedesarrollo. 1992. Diagnósticoy políticas de control por fuentes móviles en Colombia. Bogotá: MinAmbiente.

García, L., O. Rubiano. 1984. Comunidades de líquenes como indicadores de niveles de calidad del aire en Colombia. Cont Amb. 8: 73-90.

Giordani, P. 2007. Is the diversity of epiphytic lichens a reliable indicator of air pollution? A case study from Italy. Env Poll. 146: 291-576.

Hawksworth, D., T. Iturriaga, A. Crespo. 2005. Líquenes como bioindicadores inmediatos de contaminación y cambios medio-ambientales en los trópicos. Rev Iberoam Mic. 22: 71-82.

Ibagos, A. 1. 1977. Contribución al estudio de la contaminación atmosférica en la ciudad de Bogotá. Trabajo de Grado. Bogotá: Departamento de Biología, Facultad de Ciencias, Universidad Nacional de Colombia.

Jaramillo, M., L. Botero. 2010. Comunidades liquénicas como bioindicadores de calidad del aire del Valle de Aburrá. Gestión y Ambiente. 13 (1): $97-$ 110 .

Kirk, P. M., P. F. Cannon, D. W. Minter, J. A. Stalpers. 2008. Ainsworth \& Bisby's. Dictionary of the fungi. 10th ed. Wallingford: Ed. Cabi Europea.
Kricke, R., S. Loppi. 2002. Bioindication: The IAP approach. En: P. L. Nimis, C. Scheidegger, P. A. Wolseley (Eds.). Monitoring with lichensmonitoring, ichens. NATO Science Series, Vol. 7, pp. 21-37.

Lakatos M., U. Rascher, B. Budel. 2006. Citado por: Rincón Espitia, A., Aguirre J., Lucking R. Líquenes corticícolas en el Caribe colombiano. Caldasia 33 (2): 331-47.

Le Blanc, F., J De Sloover. 1970. Relation between industrialization and the distribution and growth of epiphitic lichens and mossees in Montreal. Can J Bot. 48: 1486-96.

Lijteroff, R. 2000. Diagnosis y gestión ambiental urbana en la ciudad de San Luis. Tesis de doctorado. San Luis: Universidad Nacional de San Luis.

Lijteroff, R., S. Giordano, P. Adamo, S. Sorbo, Y. Vingiani. 2005. Atmospheric trace metal pollution in the naples urban area based on results from moss and lichen bags. Env Poll. 136: 431-42.

Lücking, R. 1998. Ecology of foliicolous lichens at the «botarrama» trail (Costa Rica) a neotropical rain forest. III. Phorophyte ranges and patterns of phorophyte preferences. Phyton. 38: 195-219.

Méndez, H., J. Nájera. 2011. El uso de líquenes como biomonitores para evaluar el estado de la contaminación atmosférica a nivel mundial. Biocenosis 25 (1-2): 51-67.

Montoya Páez, J. F. 1998. Programa nacional de la contaminación por fuentes móviles. Informe Final. Bogotá: MinAmbiente.

Mosquera, Y. 2011. Líquenes como indicadores de la calidad del aire en Quibdó, Chocó, Colombia. Tesis de Grado. Quibdó: Universidad Tecnológica del Chocó

O’Neil W. B., P.G Rubio, G. Lora, F. Sánchez, E. Medina. 1992. Contaminación atmosférica por fuentes móviles. Bogotá: Departamento Nacional de Planeación, Fedesarrollo.

Rubiano, L. J., M. Chaparro. 2006. Delimitación de áreas de isocontaminación atmosférica en el campus de la Universidad Nacional de Colombia mediante el análisis de bioindicadores (líquenes epifitos). Bogotá: Facultad de Ciencias, Universidad Nacional de Colombia.

Rubiano, L. J. 1987. Delimitación de áreas de isocontaminación en Cali y Medellín utilizando líquenes como indicadores. Perez Arbelaiza. 1 (4-5): 7-41.

Rubiano, L. J. 1989. Líquenes como indicadores de contaminación en la termoeléctrica de Zipaquirá y el complejo industrial de Betania, Cundinamarca. Acta Biol Colomb. 4: 95-125.

Rubiano, L. J. 2002. Monitoría de áreas de isocontaminación en la región de influencia de la central termoeléctrica Martín del Corral utilizando líquenes como bioindicadores. Perez Arbelaiza. 13: 91-104.

Santoni, C. 2004. Evaluación de la calidad del aire mediante el uso de bioindicadores, en la localidad de Juana Koslay, San Luis, Argentina. Tesis de Licenciatura. San Luis: Universidad Nacional de San Luis.

Seinfeld, J. 1978. Contaminación atmosférica. Fundamentos físicos y químicos. Madrid: Instituto de Estudios de Administración Local. 558 pp.

Varona, G., S. Cantor, S. Urbano. 2002. Estudio de líquenes como bioindicadores de calidad de aire en la ciudad de Popayán, departamento del Cauca, Colombia. En: Ecología, Biodiversidad y Conservación. 172 pp.

Valois, H. 2006. Líquenes aproximación a su conocimiento en un bosque pluvial tropical de departamento de Chocó (Colombia). Revista Institucional, Universidad Tecnológica del Chocó 24: 8-15. 